W. M. Kantor Note on Lie algebras

\title{
Note on Lie algebras, finite groups and finite geometries
}

\author{
William M. Kantor*
}

The subject of this note began with Thompson [Th1,2]. In the course of constructing his simple group $T h$, he considered the Lie algebra $L$ over $\mathbb{C}$ of type $E_{8}$. He constructed a decomposition $L=H_{1} \perp \ldots \perp H_{31}$ using a family $\mathcal{H}=\left\{H_{1}, \ldots, H_{31}\right\}$ of Cartan algebras that are pairwise perpendicular with respect to the Killing form (he called this a "Dempwolff decomposition" of $L$; the construction was made with the assistance of $\mathrm{P}$. Smith and a computer). Moreover, $\mathcal{H}$ is closed under Lie multiplication. Thompson showed that such a decomposition is unique up to conjugacy in $E_{8}(\mathbb{C})$. Moreover, he found that the stabilizer of $\mathcal{H}$ in $E_{8}(\mathbb{C})$ has a subgroup that is a nonsplit extension of the form $2^{5} S L(5,2)$, and he constructed a lattice $\Lambda \subset L$, invariant under this group and positive definite with respect to the Killing form, such that $A$ ut $\Lambda=\mathbb{Z}_{2} \times T h$.

Kostrikin and various coworkers studied this type of situation more generally in the case of other simple complex Lie algebras $L$ [Bo], [KKU1-6], [KT1,2], [T1-3]. Their goals apparently were to look for interesting lattices and groups while at the time studying interesting properties of Lie algebras. Some aspects of what they found fit nicely into the "Groups and Geometries" framework of the present conference. This note summarizes a small part of their research, especially its relationship to questions in geometry and combinatorics.

The above papers considered a set $\mathcal{H}=\left\{H_{1}, \ldots, H_{h+1}\right\}$ of Cartan subalgebras of a simple complex Lie algebra $L$ such that $L=H_{1} \perp \ldots \perp H_{h+1}$ with respect to the Killing form, where $h$ is the Coxeter number of $L$. Such a set $\mathcal{H}$ is called an orthogonal decomposition $(O D)$ of $L$. Note that it is not difficult to find a direct sum decomposition $L=H_{1} \oplus \cdots \oplus H_{h+1}$ of $L$ into Cartan subalgebras. Bringing the Killing form into the picture greatly restricts the possibilities.

* Supported in part by the National Science Foundation.

This note was written during a term spent at Ohio State University, whose hospitality is gratefully acknowledged. 
$L$.

Let Aut $\mathcal{H}$ denote the stabilizer of $\mathcal{H}$ in the simple Lie group corresponding to

\section{Existence of $O D$ 's [Bo], [Iv1-2], [KKU1-6], [T1]}

$O D$ 's are known to exist for each of the following types of Lie algebras (where $p$ denotes any prime, $n$ is any positive integer, and $\ell$ is any integer $\geq 3): A_{p^{n}-1}, B_{\ell}$, $C_{2^{n-1}}, D_{\ell}, F_{4}, E_{6}, E_{7}, E_{8}$ and $G_{2}$. For now, let me note that there is an additional example known in the case of $E_{8}$, with $A u t \mathcal{H} \cong 5^{3} S L(3,5)$ (split) [Bo]. All $O D^{\prime} \mathrm{s}$ have been classified in the cases $A_{1}, A_{2}, A_{3}, A_{4}, C_{2}$ [KKU4]. There is more than one class of $O D^{\prime}$ s under the action of the corresponding Lie group for algebras of type $G_{2}, E_{7}, B_{\ell}$ and $D_{\ell}(\ell \geq 4)[K K U 1,2]$.

Open. Is there an $O D$ in the Lie algebras $A_{3}$ or $C_{3}$ ? In $[K K U \mathbf{1}, 3]$ it is conjectured that (i) there is an $O D$ in the case $A_{\ell}$ if and only if $\ell=p^{n}-1$ for some prime $p$ and some $n$; and (ii) there is an $O D$ in the case $C_{\ell}$ if and only if $\ell$ is a power of 2.1 )

An $O D$ is called "multiplicative" if it is closed under Lie multiplication. Multiplicative $O D^{\prime} \mathrm{s}$ exist if and only if $L$ has type $A_{1}, B_{2^{n}-1}$ or $D_{2^{n}}$ with $n \geq 2$, $E_{8}$ or $G_{2}$; moreover, all are conjugate under the action of the corresponding Lie group [Hes], [KKU2]. In each of these cases, $\mathcal{H}$ satisfies an additional condition: Aut $\mathcal{H}$ is inteducible on $L . O D^{\prime}$ s with the latter property are the ones investigated in the greatest detail in the above references ${ }^{2)}$; of course, this also fits into recent work on the subgroup structure of finite groups of Lie type. The following theorem represents the present status of classifying such $O D$ 's [KKU2,4], [KT1], [T1-3]:

THEOREM. Assume that $\mathcal{H}$ is an $O D$ such that $A$ ut $\mathcal{H}$ is irreducible on $L$. Then $L$ can be of type $A_{p^{n}-1}$ or $B_{\left[\left(p^{n}-1\right) / 2\right]}$ for any prime $p$ and any $n \geq 1, G_{2}, F_{4}, E_{6}$ or $E_{8}$; all possibulities have been classified in each of these cases. $L$ cannot be the Lite algebra $A_{\ell}$ or $B_{\ell}$ for $\ell$ not of the above form.

There is also a partial classification in the case of $D_{\ell}$ when $\mathcal{H}$ arises in a specific combinatorial manner that will be described later [Iv2]. There are also other algebras for which nonexistence of $O D^{\prime} \mathrm{s}$ is known in the irreducible case [T1].

A Jordan subgroup of Aut $L$ is an elementary abelian subgroup $J$ whose normalizer $N$ is finite and maximal among the normalizers of elementary abelian

1) This is called the "Winnie-the-Pooh problem" in [KKU3], based on a purported quote from Winnie, which the translator of [KKU3] (L. Queen, assisted by J. H. Conway) sought unsuccessfully in the Pooh books [KKU3, p. 114, footnote]. The reason for the futility of this search is indicated in [Ko, p. 180].

2) Determining them is the "weakened Winnie-the-Pooh problem" [KKU3],[T1]. 
subgroups of Aut $L$, and such that $J$ is a minimal normal subgroup of $N$. All Jordan subgroups of simple complex Lie algebras were determined in [Ale]. For every known $O D$ such that $A u t \mathcal{H}$ is transitive on $\mathcal{H}$, Aut $\mathcal{H}$ lies in such a normalizer $N$, with $J$ acting trivially on $\mathcal{H}$ (compare [Bo]). More generally, for every known $O D \mathcal{H}$, the kernel of the action of Aut $\mathcal{H}$ on $\mathcal{H}$ is "large".

The case $A_{\ell}$ of the theorem is settled using the classification of finite 2-transitive permutation groups. These arise as follows. Assume that Aut $\mathcal{H}$ is irreducible on $L$. Then the stabilizer $(A u t \mathcal{H})_{H_{1}}$ must be irreducible on $H_{1}$. However, the normalizer of $H_{1}$ in the Lie group $S L(\ell+1, \mathbb{C})$ induces the Weyl group $S_{\ell+1}$ on $H_{1}$. The group induced by $(\text { Aut } \mathcal{H})_{H_{1}}$ on $H_{1}$ occurs within the irreducible representation of degree $\ell$ of $S_{\ell+1}$. It follows that the action of $\left(\right.$ Aut $\mathcal{H}_{\mathrm{H}_{1}}$ within $S_{\ell+1}$ must be 2-transitive. Of course, this greatly limits this group and hence also Aut $\mathcal{H}$.

My interest in $O D$ 's stems from the geometry and combinatorics involved in the construction of the known examples. Therefore, the remainder of this note will focus on constructions.

\section{Constructions within the Lie algebra $A_{p^{n}-1}$}

Consider the Lie algebra $L=\operatorname{sl}\left(p^{n}, \mathbb{C}\right)$ consisting of all $p^{n} \times p^{n}$ matrices of trace 0 , where $p$ is a prime and $n \geq 1$. Let $V=\mathbb{F}_{p}^{2 n}$, let $\varepsilon$ be a primitive complex $p$ th root of 1 , and define $p \times p$ matrices $D$ and $P$ as follows:

$$
D=\operatorname{diag}\left(1, \varepsilon, \ldots, \varepsilon^{p-1}\right) \text { and }
$$

$P$ is the permutation matrix corresponding to the $p$-cycle $(1, \ldots, p)$.

Both of these have trace 0 . With each $u=\left(a_{1}, b_{1}, \ldots, a_{n}, b_{n}\right) \in V-\{0\}$ associate the matrix

$$
J_{u}=D^{a_{1}} P^{b_{1}} \otimes \cdots \otimes D^{a_{n}} P^{b_{n}} \in L \text {. }
$$

These $p^{2 n}-1$ matrices $J_{t}$ form a basis of $L$. The commutator of the matrices $J_{t}$ and $J_{v}$ is $\varepsilon^{(u, v)} I$, where, for $u=\left(a_{1}, b_{1}, \ldots, a_{n}, b_{n}\right)$ and $v=\left(a_{1}^{\prime}, b_{1}^{\prime}, \ldots, a_{n}^{\prime}, b_{n}^{\prime}\right)$, $(u, v)=\Sigma_{i}\left(a_{i} b_{i}^{\prime}-a_{i}^{t} b_{i}\right)$ defines a nonsingular alternating bilinear form on $V$. (We will soon see that there is an extraspecial group lurking here.) Moreover, $\operatorname{Tr}\left(J_{u} J_{v}\right)=0$ if $u+v \neq 0$ : in this case $J_{u}$ and $J_{v}$ are perpendicular.

All known $O D$ 's of Lie algebras of type $A_{\ell}$ have $\ell=p^{n}-1$ for some $p$ and $n$ as above, and, up to equivalence under the action of $G L\left(p^{n}, \mathbb{C}\right)$, arise as $L=$ $H_{1} \perp \ldots \perp H_{p^{n}+1}$ with each $H_{i}$ spanned by some of the $J_{u}$ 's. In this case, let $V_{i}=\left\{0, u \mid J_{i} \in H_{i}\right\}$. Then

$$
\begin{aligned}
& \left|V_{i}\right|-1=\operatorname{dim} H_{i}=p^{n}-1, \\
& V_{i} \text { is a subspace of } V, \text { and } \\
& \left(V_{i}, V_{i}\right)=0 .
\end{aligned}
$$

Therefore, the $V_{i}$ s form a spread $\Sigma$ in $V$ : a collection of $p^{n}+1 n$-spaces of the $2 n$-space $V$ such that each nonzero vector is in a unique member of $\Sigma$. Moreover, 
this is a symplectic spread: there is a symplectic structure on $V$ such that each member of $\Sigma$ is totally isotropic [Iv1].

Conversely, each symplectic spread of $V$ produces an $O D$ by the previous recipe. Symplectic spreads were studied for use in finite geometry and coding theory (cf. $[\mathbf{K a 1}, 2]$ ) several years before this connection arose with Lie algebras [Iv1].

\section{Examples of symplectic spreads}

Clearly, there is no need to restrict the base field $K$ to $\mathbb{F}_{p}$ : this could be any field whatsoever, but we are only interested in the case in which $K$ is a finite field $\mathbb{F}_{q}$. Then each spread consists of $q^{n}+1 n$-spaces in a $2 n$-space over $\mathbb{F}_{q}$. The importance of spreads is that they produce affine planes: Let $\mathcal{A}(\Sigma)$ denote the point-line geometry whose points are vectors and whose lines are the cosets $W+v$ with $W \in \Sigma, v \in V$. Then $\mathcal{A}(\Sigma)$ is an affine plane of order $q^{n}$ : any two points are on a unique line; given a line $L$ and a point $v$ not on it, there is a unique line through $v$ disjoint from $L$; and each line has exactly $q^{n}$ points. There is an obvious notion of parallelism, and by adjoining a new "line at infinity" that "contains" all parallel classes one obtains a projective plane (of order $q^{n}$ ). For each $c \in V$ the translation $v \mapsto v+c$ is an automorphism fixing every parallel class. These affine planes (and their associated projective planes) are called translation planes. The nontrivial translations are just the automorphisms of $\mathcal{A}(\Sigma)$ that fix every parallel class but fix no point. The group of translations will be identified with $V$.

EXAMPLE 1. If $V$ is a 2-dimensional vector space over $K$, its set $\Sigma$ of 1 -spaces is a spread, producing the desarguesian plane $\mathcal{A}(\Sigma)$ of order $q$.

These 1-spaces are totally isotropic with respect to any nonsingular alternating bilinear form $\left(\right.$, ) on the $K$-space $V$. If $K^{\prime}$ is any subfield of $K$, and if $\operatorname{Tr}: K \rightarrow K^{\prime}$ is the trace map, then $\Sigma$ becomes a spread of the $K^{\prime}$-space $V$ consisting of totally isotropic subspaces with respect to the alternating $K^{\prime}$-bilinear form $\operatorname{Tr}($, ). The resulting affine plane is identical to the one obtained using $K$. Nevertheless, this yields an example of a symplectic spread for every prime power $p^{n}$.

This suggests that it matters what fields are used. The kernel of any spread $\Sigma$, or of the plane $\mathcal{A}(\Sigma)$, is the set of endomorphisms of the group $V$ of translations that send each member of $\Sigma$ into itself. This is a ring under the obvious operations, and is in fact a field. Thus, it is fairly standard to view a translation plane as a vector space over its kernel, rather than over a subfield of its kernel. Any isomorphism between two translation planes must send the translation group of the first plane to the translation group of the second, and (after the kernels are identified) is a semilinear transformation over the kernel. This also yields a natural notion of equivalence of spreads - under the action of the group $\Gamma L(V)$.

Example 2. Let $F$ be a field of odd order, let $s \in F$ be a nonsquare, let $1 \neq$ 
$\sigma \in$ Aut $F$ with fixed field $K$, and let $\rho \in$ Aut $F$. View $F^{2}$ as a $K$-space. Then the subspaces

$$
\begin{aligned}
& \{(0, y) \mid y \in F\} \text { and } \\
& \left\{\left(x, m x-s m^{\rho} x^{\sigma}\right) \mid y \in F\right\} \text { for } m \in F
\end{aligned}
$$

form a spread. $\mathcal{A}(\Sigma)$ is called a "twisted field plane"; it was discovered by Albert [Al], who showed it is nondesarguesian.

In odd characteristic, there is (up to equivalence) only one known infinite family of symplectic spreads of dimension $>2$ over their kernels: when $s=-1$ (so $|F| \equiv 3(\bmod 4))$ and $p=\sigma$, the above example is symplectic with respect to the alternating form $\left(\left(x_{1}, y_{1}\right),\left(x_{2}, y_{2}\right)\right)=\operatorname{Tr}\left(x_{1}^{p} y_{2}-x_{2}^{p} y_{1}\right)$, where $\operatorname{Tr}: F \rightarrow K$ is the trace map.

Example 3. Let $K$ be a field of odd order, let $s \in K$ be a nonsquare, and let $\sigma \in$ Aut $K$. Define a binary operation * on $K^{2}$ by

$$
(a, b) *(u, v)=\left(a v+b u, a s u^{\sigma}+b v\right) \text {. }
$$

(This produces $\mathbb{F}_{q^{2}}$ if and only if $\sigma=1$.) View $K^{2} \times K^{2}$ as a left vector space over $K$. Then the subspaces

$$
\begin{aligned}
& \left\{(0, y) \mid y \in K^{2}\right\} \text { and } \\
& \left\{(x, x * m) \mid x \in K^{2}\right\}, \text { for } m \in K^{2},
\end{aligned}
$$

form a spread that is symplectic with respect to the alternating form

$$
\left(\left(x_{1}, x_{2}, x_{3}, x_{4}\right),\left(y_{1}, y_{2}, y_{3}, y_{4}\right)\right)=x_{1} y_{4}-x_{4} y_{1}+x_{2} y_{3}-x_{3} y_{2} \text {. }
$$

ExAMPLE 4. Hering [He] defined a subgroup $G \cong S L(2,13)$ of $S L(6,3)$, and a subspace $W$, and showed that $\Sigma=\left\{W^{g} \mid g \in G\right\}$ is a spread. The group $V \times G$ is 2-transitive on the points of $\mathcal{A}(\Sigma)$. In fact, $G<S_{p}(6,3)$, and $W$ is totally isotropic, so that $\Sigma$ is symplectic.

The $O D$ 's for algebrus of type $A_{\ell}$ in the Theorem arise from spreads appearing in Examples 1 and 4. In fact, these spreads are just those for which the automorphism group of the corresponding plane $\mathcal{A}(\Sigma)$ is 2-transitive on the points of the plane.

EXAMPLE 5. Two families of symplectic spreads in $\mathbb{F}_{q}^{4}$, with $q \geq 27$ a power 3 , are given in $[\mathbf{K a 2}]$ and $[\mathbf{P T}]$. The first of these arises from the Ree groups ${ }^{2} G_{2}\left(3^{2 e+1}\right)$.

Of course, for the above examples subfields of $K$ can be used to produce more symplectic spreads. With this proviso, every known symplectic spread in odd characteristic appears in Examples 1-5.

EXAMPLE 6. The Suzuki group $S z(q), q=2^{2 e+1}>2$, acts on an ovoid in $\mathbb{F}_{q}^{4}$. Applying the graph automorphism of $S p(4, q)$ produces a symplectic spread in $\mathbb{F}_{q}^{4}$ 
(cf. [L $\mathbf{u}]$ ). In fact, when $q$ is even, the only known symplectic spreads in $\mathbb{F}_{q}^{4}$ are the desarguesian one and this one.

EXAMPLE 7. Large numbers of symplectic spreads are constructed in [Ka1] when $q$ is even and $n$ is odd. Here is one especially interesting family.

Let $K=\mathbb{F}_{q}$ and $F=\mathbb{F}_{q^{2 n}}$ with $q$ even and $n$ odd. Let $\operatorname{Tr}: \mathbb{F}_{q^{n}} \rightarrow K$ be the trace map, and let $W$ be its kernel. Finally, let $r \in \mathbb{F}_{q^{2}}-\mathbb{F}_{q} \subseteq F$ and let $s \in F$ have order $q^{n}+1$. Then $\Sigma=\left\{s^{i}(W+r K) \mid 0 \leq \dot{i}<q^{n}+1\right\}$ is a spread. Moreover, it is symplectic with respect to the alternating form $(u, v)=\operatorname{Tr}(u \bar{v}+v \bar{u})$; for, $z \mapsto s z$ preserves this form, $\left(\mathbb{F}_{q^{n}}, \mathbb{F}_{q^{n}}\right)=0$, and $(r, w)=\operatorname{Tr}(r w+\bar{r} w)=(r+\bar{r}) \operatorname{Tr}(w)=0$ for $w \in W$.

The proof that $\Sigma$ is a spread is not difficult (cf. [Ka1]). The automorphism group of $\mathcal{A}(\Sigma)$ has a subgroup $\left\{z \mapsto s^{i} z+c \mid 0 \leq i<q^{n}+1\right.$ and $\left.c \in F\right\}$ that is transitive on lines, in fact, regular on the set of incident point-line pairs (sharply flag-transitive). The planes are nondesarguesian, and are the only known nondesarguesian affine planes of even order having a sharply flag-transitive automorphism group.

\section{Open problems}

1. Find more examples of symplectic spreads.

2. It appears to be very difficult to determine whether a more or less "random" spread is symplectic when $\operatorname{dim} V>4$.

3. What effect does "symplecticness" have on the internal geometric properties of the affine plane $\mathcal{A}(\Sigma)$ ?

\section{Constructions within the Lie algebra $C_{2^{n-1}}$}

View $\mathbb{C}^{2^{n}}$ as $\mathbb{C}^{2} \otimes \cdots \otimes \mathbb{C}^{2}$ (all tensor products will have $n-1$ factors). Let $D$, $P$ and $J_{u}$ (with $u \in \mathbb{F}_{2}^{2 n-2}-\{0\}$ ) be as in the case of $A_{2^{n}-1}$ (with $p=2$ ), and equip $\mathbb{C}^{2^{n}}$ with the nonsingular alternating bilinear form whose matrix is $S=$ $I \otimes \cdots \otimes I \otimes(D P)$. We may assume that $L=\left\{M \in g l\left(2^{n}, \mathbb{C}\right) \mid S^{-1} M^{t} S=-M\right\}$.

Note that $Q=\langle D, P\rangle \otimes \cdots \otimes\langle D, P\rangle \otimes\langle i D, D P\rangle$ is an extraspecial group of isometries of $S$ of order $2^{2 n-1}$ (i.e., it is a subgroup of $S p\left(2^{n}, \mathbb{C}\right.$ ) that is the central product of $n-2$ dihedral groups and a quaternion group, all of order 8 ). Its center $Z(Q)$ is $\langle-I\rangle$ and will be identified with $\mathbb{F}_{2}$, while $Q / Z(Q)$ will be identified with $\mathbb{F}_{2}^{2 n-2}$. There is a nonsingular quadratic form $\varphi: Q / Z(Q) \rightarrow \mathbb{F}_{2}$ such that $\varphi(x Z(Q))=x^{2} \in Z(Q)$; its associated skew-symmetric bilinear form, defined by $(x Z(Q), y Z(Q))=[x, y]$, is the same one used earlier for $A_{2^{n}-1}$. Moreover, $S^{-1} J_{u}^{t} S=(-1)^{\varphi(u)} J_{u}$, and $\left\{J_{u} \mid \varphi(u)=1\right\}$ is a basis of $L$.

If $\mathcal{H}=\left\{H_{j}\right\}_{j}$ is an $O D$ of the Lie algebra $A_{2^{n}-1}$ obtained using matrices $J_{u}$ as above, then $\left\{H_{j} \cap L\right\}_{j}$ is an $O D$ of the present algebra $L$. The totally isotropic 
$(n-1)$-spaces of $\mathbb{F}_{2}^{2 n-2}$ associated with $\mathcal{H}$ are still totally isotropic, but they are not totally singular with respect to $\varphi$. Nevertheless, we see that any symplectic spread of $\mathbb{F}_{2}^{2 n-2}$ gives rise to an $O D$ of $L$ [Bo] (cf. [KKU1, p. 294]). Moreover, this discussion helps to clarify the remark made earlier that there was an extraspecial group lurking behind the $A_{p^{n}-1}$ construction.

\section{Constructions within the Lie algebra $D_{\ell}$}

This Lie algebra $L$ can be identified with the space of all skew-symmetric $2 \ell \times 2 \ell$ matrices over $\mathbb{C}$. If $E_{i j}$ denotes the $2 \ell \times 2 \ell$ matrix with $i j$-entry 1 and all others 0 , and if $E(i, j)=E_{i j}-E_{j i}$, then $\{E(i, j) \mid 1 \leq i<j \leq 2 \ell\}$ is a basis of $L$. There is a natural way to obtain an $O D$ given in [KKU6]. A parallel class of the set $X=\{1, \ldots, 2 \ell\}$ is a partition $\pi$ of $X$ into $\ell$ pairs; a parallelssm of $X$ is a partition $\Pi$ of the set of 2-element subsets of $X$ into $2 \ell-1$ parallel classes [Ca]. Then $\mathcal{H}=\left\{H_{\pi} \mid \pi \in \Pi\right\}$ is an $O D$ of $L$, where $H_{\pi}$ is the subspace spanned by $\{E(i, j) \mid\{\dot{i}, j\} \in \pi\}$. So there are quite a lot of $O D^{\prime} \mathrm{s}$ of these Lie algebras.

ExAMPLE 1. Let $2 \ell=2^{n}$, identify $\{1, \ldots, 2 \ell\}$ with $\mathbb{F}_{2}^{n}$, and let $\Pi$ be the set of parallel classes of lines of the affine space $A G(n, 2)$. Explicitly, for $a \in \mathbb{F}_{2}^{n}-\{0\}$ let $H_{a}$ be the subspace spanned by $\{E(u, v) \mid v=u+a\}$. This produces a multiplicative $O D$ since $\left[H_{a}, H_{b}\right]=H_{a+b}$ for all distinct nonzero $a, b \in \mathbb{F}_{2}^{n}$.

EXAMPLE 2. Let $G$ be any group of order $2 \ell-1$. Identify $X$ with $\{0\} \cup G$. Let $\Pi=\left\{\pi_{a} \mid a \in G\right\}$, where $\pi_{a}=\{0, a\} \cup\left\{\left\{x a, x^{-1} a\right\} \mid 1 \neq x \in G\right\}$. This defines a parallelism, and $G$ acts on both $X$ and $\Pi$ in an obvious manner (and is even transitive on $\Pi$ ). The resulting $O D^{\prime} \mathrm{s}$ are studied at length in [KKU6].

These examples represent a negligible proportion of the parallelisms of a $2 \ell-$ set. See [Ca, pp. 64-67] for asymptotic estimates of the number of inequivalent parallelisms.

There is an obvious diagonal group underlying $\mathcal{H}$ for any $\Pi$ : the subgroup of $G L(2 \ell, \mathbb{C})$ consisting of all diagonal matrices with \pm 1 on the diagonal induces a group $\mathbb{Z}_{2}^{2 t}-1$ of diagonal transformations of $L$ with respect to the basis consisting of the $E(i, j)$ 's (once again $-1 \in G L(2 \ell, \mathbb{C}$ ) induces the identity on $L$ ). The stabilizer Aut $\Pi$ of $\Pi$ in $S_{2 \ell}$ also acts on $\mathcal{H}$. If $\ell \geq 4$ and $\mathcal{H}$ is an $O D$ arising from a parallelism $\Pi$, then $A$ ut $\mathcal{H}$ is irreducible on $L$ if and only if one of the following holds [Iv2]:

$$
\begin{aligned}
& 2 \ell=2^{n}, \Pi \text { is as in Example } 1 ; \\
& 2 \ell=12, \text { Aut } \Pi \cong \operatorname{PSL}(2,11) ; \text { or } \\
& 2 \ell=28, \text { Aut } \Pi \cong{ }^{2} G_{2}(3) .
\end{aligned}
$$

This is proved using the classification of finite 2-transitive groups. 


\section{Further constructions}

There is a similar construction for the algebra $L$ of type $B_{\ell}$. Namely, this time $L$ can be viewed as the set of all skew-symmetric $(2 \ell-1) \times(2 \ell-1)$ matrices over $\mathbb{C}$, so just restrict the preceding types of $O D$ 's for $D_{\ell}$ to the matrices whose last row and column consist entirely of 0 's. Moreover, in [T2] it is shown that any $O D \mathcal{H}$ of an algebra of type $B_{\ell}$ must have this form if Aut $\mathcal{H}$ is irreducible on $L$.

There is also an entirely different construction of $O D$ 's for Lie algebras of type $D_{2^{n-1}}$, one very similar to that used for type $C_{2^{n-1}}$ [Bo] (cf. [KKU1, p. 294]).

The following general construction technique for an $O D$ in any $L$ is given in [KKU2]: Let $\Phi$ be the root system, $\Phi^{+}$the set of positive roots with respect to some ordering, and $L=H_{0} \oplus \bigoplus_{\alpha \in \Phi} \mathbb{C} x_{\alpha}$ a Cartan decomposition relative to the Cartan subalgebra $H_{0}$. Assume that $\mathcal{H}=\left\{H_{0}, \ldots, H_{h}\right\}$ is an $O D$ and that, for $0<i \leq h$, the Cartan subalgebra $H_{i}$ is spanned by elements of the form $x_{\alpha}+c_{\alpha} x_{-\alpha}$ with $\alpha \in \Phi^{+}$and $c_{\alpha} \in \mathbb{C}^{*}$. Let $\Phi_{i}$ be the set of positive roots $\alpha$ associated in this manner with $H_{i}$. Then $\Phi^{+}$is partitioned by the sets $\Phi_{i}$, and $\alpha, \beta \in \Phi_{i} \Rightarrow \alpha+\beta$, $\alpha-\beta \notin \Phi ;$ conversely, every partition of $\Phi^{+}$into $h$ sets $\Phi_{i}$ having this property produces an $O D$.

In [KKU2] it is show that the only simple Lie algebras $L$ admitting $O D$ 's arising in this manner are those of type $A_{1}, B_{2 \ell-1}$ or $D_{2 \ell}$ with $\ell \geq 2, E_{7}, E_{8}$ and $G_{2}$. This result is used, in turn, to determine all multiplicative $O D$ 's.

EXAMPLE. If $\Phi$ has type $G_{2}$ and $\alpha_{1}, \alpha_{2}$ is a base, use the partition $\left\{\alpha_{1}, 3 \alpha_{1}+2 \alpha_{2}\right\}$, $\left\{\alpha_{1}+\alpha_{2}, 3 \alpha_{1}+\alpha_{2}\right\},\left\{2 \alpha_{1}+\alpha_{2}, \alpha_{2}\right\}$. The resulting $O D$, with Aut $\mathcal{H} \cong 2^{3} S L(3,2)$ (nonsplit), is studied in detail in [KKU5].

As remarked earlier, part of the original motivation for the study of $O D$ 's was the search for interesting lattices: ones invariant under all or most of Aut $\mathcal{H}$. See Kostrikin"s survey [Ko] for indications of this, together with pointers towards further literature.

\section{References}

[Al] A. A. Albert, On the collineation groups associated with twisted fields, Calcutta Math. Soc. Golden Jubilee Commemoration volume (1958/59), part II, $485-497$.

[Ale] A. V. Alekseevskii, Jordan finite commutative subgroups of simple complex Lie groups, Functional Anal. Appl. 8 (1974), 277-279. 
[BKL] L. Bader, W. M. Kantor and G. Lunardon, Symplectic spreads from twisted fields (to appear).

[Bo] A. V. Borovik, Jordan subgroups and orthogonal decompositions, Algebra and Logic 28 (1990) $248-254$.

[Ca] P. J. Cameron, Parallelisms of complete designs. LMS Lectures Notes 23, Cambridge University Press, Cambridge 1976.

[He] C. Hering, Eine nicht-desarguessche zweifach transitive affine Ebene der Ordnung 27, Abh. Math. Sem. Hamb. 34 (1969) 203-208.

[Hes] W. H. Hesselink, Special and pure gradings of Lie algebras, Math. Z. 179 (1982) 134-149.

[Iv1] D. N. Ivanov, Orthogonal decompositions of Lie algebras of type $A_{p^{n}-1}$, and isotropic fiberings, Russian Math. Surveys 42 (1987) 141-142.

[Iv2] D. N. Ivanov, Orthogonal decompositions of Lie algebras of types $A_{p^{n}-1}$ and $D_{n}$ with a finite number of classes of similar invariant sublattices, Moscow U. Math. Bull. 44 (1989) 59-64.

[Ka1] W. M. Kantor, Spreads, translation planes and Kerdock sets I, II, SIAM J. Alg. Disc. Meth. 3 (1982) 151-165, 308-318.

[Ka2] W. M. Kantor, Ovoids and translation planes, Can. J. Math. 34 (1982) 11951207.

[Ko] A. I. Kostrikin, Invariant lattices in Lie algebras and their automorphism groups, Group Theory, Proc. Singapore Conf. 1987 (eds. K. N. Cheng and Y. K. Leong), de Gruyter 1989, 171-181.

[KKU1] A. I. Kostrikin, I. A. Kostrikin, and V. A. Ufnarovski, Orthogonal decompositions of simple Lie algebras, Soviet Math. Dokl. 24 (1981), 292-296.

[KKU2] A. I. Kostrikin, I. A. Kostrikin, and V. A. Ufnarovski, Multiplicative decompositions of simple Lie algebras, Soviet Math. Dokl. 25 (1982) 23-27.

[KKU3] A. I. Kostrikin, I. A. Kostrikin, and V. A. Ufnarovski, Orthogonal decompositions of simple Lie algebras (type $A_{n}$ ), Proc. Steklov Inst. Math. 158 (1983) 113-129.

[KKU4] A. I. Kostrikin, I. A. Kostrikin, and V. A. Ufnarovski, On the uniqueness of orthogonal decomposotions of Lie algebras of types $A_{n}$ and $C_{n}$, I, II. Mat. Issled. 74 (1983) 80-105, 106-116 (Russian).

[KKU5] A. I. Kostrikin, I. A. Kostrikin, and V. A. Ufnarovski, Invariant lattices of type $G_{2}$ and their automorphism groups, Proc. Steklov Inst. Math. 165 (1985) $85-105$.

[KKU6] A. I. Kostrikin, I. A. Kostrikin, and V. A. Ufnarovski, On decompositions of classical Lie algebras, Proc. Steklov Inst. Math. 166 (1986) 117-133. 
[KT1] A. I. Kostrikin and P. H. Tiep [F. K. T'ep] ${ }^{3)}$, Classification of irreducible orthogonal decompositions of simple Lie algebras of type $A_{n}$, St. Petersburg Math. J. 3 (1992) 571-593.

[KT2] A. I. Kostrikin and P. H. Tiep, Orthogonal decompositions and integral lattices (de Gruyter, to appear).

[Lï] H. Lüneburg, Translation planes. Springer-Verlag, Berlin 1980.

[PT] S. E. Payne and J. A. Thas, Spreads and ovoids in finite generalized quadrangles (submitted).

[T1] P. H. Tiep [F. K. T'ep], Irreducible orthogonal decompositions in Lie algebras, Math. USSR Sbornik 68 (1991) 257-275.

[T2] P. H. Tiep, A classification of irreducible orthogonal decompositions of the simple complex Lie algebras of type $B_{n}$, Comm. in Algebra 19 (1991) 2729 2775.

[T3] P. H. Tiep, A characteristic property of the multiplicative orthogonal decomposition of the Lie algebra $D_{4}$, Moscow U. Math. Bull, 45 (1990) 46-49.

[Th1] J. G. Thompson, A conjugacy theorem for $E_{8}$, J. Algebra 38 (1976) 525-530.

[Th2] J. G. Thompson, A simple subgroup of $E_{8}(3)$, Finite Groups (Proc. Intl. Symp. Sapporo 1974; ed. N. Iwahori), Tokyo 1976, 113-116.

3) The author has remarked to me concerning his Vietnamese name that it "is wrong that my name is translated from Russian to English as Fam Khyu T'ep, in fact this should be Pham Huu Tiep". The incorrect translation is included in the present references only when it was used in the published English version. 